\section{Capacidade funcional e uso de serviços de saúde por idosos da Região Metropolitana de Belo Horizonte, Minas Gerais, Brasil: um estudo de base populacional}

\author{
Disability and use of health services by the elderly \\ in Greater Metropolitan Belo Horizonte, Minas \\ Gerais State, Brazil: a population-based study
}

Camila Bruno Fialho 1

Maria Fernanda Lima-Costa 2,3

Karla Cristina Giacomin 2,3

Antônio Ignácio de Loyola Filho 2,4

\footnotetext{
${ }^{1}$ Centro de Pesquisas René Rachou, Fundação Oswaldo Cruz, Belo Horizonte, Brasil. 2 Núcleo de Estudos em Saúde Pública e Envelhecimento, Fundação Oswaldo Cruz/Universidade Federal de Minas Gerais, Belo Horizonte, Brasil.

3 Faculdade de Medicina Universidade Federal de Minas Gerais, Belo Horizonte, Brasil

${ }^{4}$ Escola de Enfermagem, Universidade Federal de Minas Gerais, Belo Horizonte, Brasil.

Correspondência A. I. Loyola Filho Núcleo de Estudos em Saúde Pública e Envelhecimento, Fundação Oswaldo Cruzl Universidade Federal de Minas Gerais.

Av. Augusto de Lima 1715, 6 o andar, Belo Horizonte, $M G$ 30190-003, Brasil. aloy@cpqrr.fiocruz.br
}

\begin{abstract}
This study focused on the association between disability and use of health services among elderly individuals in Greater Metropolitan Belo Horizonte, Minas Gerais State, Brazil. The study included 1,624 elderly patients ( $\geq 60$ years) selected by representative sampling. The dependent variable was use of health services, based on three descriptors: number of physician visits, home consultations, and hospitalizations. The target independent variable was disability, including difficulty in performing activities of daily living $(A D L)$ and instrumental activities of daily living (IADL). IADL was only associated with hospitalization ( $P R=1.62$; 95\%CI: 1.16-2.26), while ADL was associated with hospitalization $(P R=1.73$; 95\%CI: 1.24-2.42) and home consultations ( $P R=$ 8.54; 95\%CI: 4.22-17.27). The findings show increased use of health services (especially more costly ones) among older adults with disabilities, and that functional health dimensions have not oriented health services, still largely conditioned on the presence of diseases.
\end{abstract}

Health Services; Frail Elderly; Aged

\section{Resumo}

O presente trabalho investigou a associação entre a capacidade funcional e utilização de serviços de saúde entre idosos residentes na Região Metropolitana de Belo Horizonte, Minas Gerais, Brasil. Participaram do estudo 1.624 idosos $(\geq 60$ anos) selecionados por meio de amostra probabilística. A variável dependente foi a utilização de serviços de saúde, baseada em três descritores: número de consultas médicas, consultas domiciliares e hospitalizações. A variável independente de interesse foi a capacidade funcional, medida pelo relato de dificuldade em realizar atividades instrumentais (AIVD) e básicas ( $A B V D)$ de vida diária. A primeira esteve associada apenas à hospitalização $(R P=1,62$; IC95\%: 1,16-2,26) $e$ a segunda apresentou-se associada à hospitalização (RP = 1,73; IC95\%: 1,24-2,42) e à consulta domiciliar ( $R P=8,54$; IC95\%: 4,22-17,27). Os resultados mostraram maior utilização de serviços de saúde, sobretudo aqueles mais onerosos, entre idosos com incapacidade funcional, e que a dimensão funcional não tem sido balizadora da atuação dos serviços, ainda condicionada à presença de doenças.

Serviços de Saúde; Idoso Fragilizado; Idoso 


\section{Introdução}

O rápido envelhecimento da população brasileira 1 acarreta importantes modificações não apenas na estrutura etária da população, mas também no seu perfil epidemiológico, configurando um quadro em que as doenças crônicas não transmissíveis (DCNT), típicas da modernidade, passam a predominar em substituição às doenças transmissíveis ${ }^{2}$. Entre as complicações das DCNT com maiores repercussões para o indivíduo e a sociedade encontra-se a incapacidade funcional, definida por Verbrugge \& Jette ${ }^{3}$ como a dificuldade em realizar atividades em qualquer domínio da vida.

Os domínios da incapacidade funcional mais investigados são as atividades básicas de vida diária (ABVD), relacionadas ao autocuidado e à sobrevivência (como tomar banho e alimentarse), e as atividades instrumentais da vida diária (AIVD), de maior complexidade e relacionadas à vida independente em comunidade (como fazer compras e utilizar o transporte). Elas podem ser avaliadas separada ou conjuntamente 4,5 . Em estudos epidemiológicos, usualmente, a incapacidade funcional é avaliada com base no autorrelato do grau de dificuldade, da necessidade de ajuda de outra pessoa ou ainda da impossibilidade de realizar as ABVD e/ou as AIVD 3. Pode-se mensurar a gravidade da incapacidade estabelecendo uma hierarquia de categorias baseadas no tipo de atividade (a incapacidade para ABVD é mais grave que a incapacidade para AIVD) 6 , ou num quantitativo de limitações para as distintas atividades 7,8 .

Entre idosos, a maior prevalência de incapacidade, bem como de doenças crônicas e comorbidades, torna a demanda por cuidados de saúde dessa população diferenciada em relação àquela apresentada por outros segmentos etários ${ }^{9}$. Os idosos utilizam mais frequente e intensamente os serviços de saúde, exigindo ações de maior complexidade e custo 1,2,10. Por exemplo, quando comparados aos jovens, os idosos são hospitalizados com maior frequência, apresentam tempo médio de permanência hospitalar maior e possuem um índice de (re)internações mais elevado 11 .

Há indícios de que a intensidade na utilização de serviços de saúde por parte de idosos com capacidade funcional prejudicada possa variar em função da gravidade da incapacidade. Estudos internacionais têm mostrado que pessoas com incapacidade grave utilizam mais os serviços de saúde em comparação àqueles com incapacidade mais leve ou sem incapacidade, seja em relação à consulta médica 12, à hospitalização, ou aos maiores custos com os serviços primá- rios de atenção à saúde 13 . No Brasil, vários estudos 6,8,14 têm investigado os fatores associados à incapacidade funcional entre idosos; contudo são raros aqueles que o fizeram quanto ao impacto da incapacidade funcional no uso de serviços de saúde. Um estudo conduzido em municípios das regiões Sul e Nordeste do Brasil mostrou que, entre idosos portadores de doenças crônicas, a prevalência de consultas médicas foi $30 \%$ mais alta entre aqueles com algum nível de incapacidade, em comparação às suas contrapartes sem limitações funcionais ${ }^{9}$.

O presente estudo epidemiológico de base populacional objetivou investigar o padrão de associação entre a capacidade funcional e a utilização de serviços de saúde entre idosos residentes na Região Metropolitana de Belo Horizonte, Minas Gerais, Brasil, em 2003, com ênfase nas consultas médicas, nas consultas médicas domiciliares e nas hospitalizações ocorridas nos 12 meses precedentes.

\section{Metodologia}

\section{Área e população de estudo}

A presente investigação foi desenvolvida na Região Metropolitana de Belo Horizonte, constituída por cerca de vinte municípios, totalizando uma população de 4,4 milhões de habitantes. O inquérito domiciliar foi realizado entre maio e junho de 2003, por meio de entrevista face a face, como suplemento da Pesquisa de Emprego e Desemprego (PED) da Fundação João Pinheiro, órgão do governo do Estado de Minas Gerais. Informações mais detalhadas podem ser obtidas em outra publicação 15 .

Os participantes do inquérito domiciliar de saúde foram selecionados por intermédio de amostra probabilística por conglomerado, em dois estágios, sendo o setor censitário do Instituto Brasileiro de Geografia e Estatística (IBGE) a unidade primária de seleção e o domicílio, a unidade amostral. O delineamento amostral objetivou selecionar uma amostra que fosse representativa da população residente nos municípios que compõem a Região Metropolitana de Belo Horizonte. Todos os moradores do domicílio amostrado, com idade igual ou superior a 20 anos e de ambos os sexos $(n=13.701)$, foram selecionados para o inquérito, e desses, todos os moradores com 60 anos ou mais $(n=1.777)$ foram elegíveis para o estudo ${ }^{15}$. Participaram do estudo os $1.624(91,4 \%)$ idosos com informações completas acerca da capacidade funcional e/ou do uso de serviços de saúde. 


\section{Variáveis do estudo}

As variáveis dependentes do estudo foram três descritores de utilização de serviços de saúde: número de consultas médicas, número de consultas domiciliares e número de hospitalizações, considerando os 12 meses anteriores à entrevista.

A variável independente de interesse foi a incapacidade funcional para ABVD e para AIVD. As ABVD contempladas no estudo foram: tomar banho, alimentar-se, vestir-se, levantar-se da cama caminhar de um cômodo ao outro no domicílio e ir ao banheiro. No tocante às AIVD, foram incluídas: fazer compras, realizar tarefas domésticas leves, preparar seu próprio alimento, tomar seus remédios e administrar o próprio dinheiro. Os participantes foram indagados quanto ao grau de dificuldade para a realização de cada uma das atividades acima descritas, considerando-se quatro opções de resposta (nenhuma; alguma; muita; só com ajuda de outra pessoa). De acordo com a resposta, foram constituídos três grupos: (1) sem limitação para AIVD e ABVD, definido pela ausência de dificuldades para realizar todas as atividades consideradas; (2) com limitação somente para AIVD, definido pelo relato de alguma dificuldade para realizar uma ou mais AIVD e de nenhuma dificuldade para realizar todas as ABVD; (3) com limitação para ABVD, definido pelo relato de alguma dificuldade para realizar uma ou mais ABVD. Todos os participantes que relataram dificuldade para pelo menos uma ABVD, também o fizeram para pelo menos AIVD, sendo incluídos na última categoria.

O modelo de Andersen 16, empregado em investigações relacionadas à utilização de serviços de saúde 17 , foi adotado para a seleção das potenciais variáveis de confusão do estudo, considerando-se três dimensões determinantes do uso de serviços de saúde: a predisposição, a capacitação e a necessidade. Neste trabalho, as características predisponentes incluíram o sexo, a faixa etária (60-69; 70-79; 80+ anos) e a situação conjugal [casado(a)/união consensual; não casado]; as características capacitantes consideradas foram o número de anos completos de escolaridade $(0-3 ; 4-7 ; 8+)$ e a filiação a plano privado de saúde (não; sim); e as características de necessidade incluíram o número de doenças crônicas $(0 ; 1 ; 2+)$ e a autoavaliação em saúde (muito boa/ boa; razoável; ruim/muito ruim). Na definição do número de doenças crônicas, considerou-se o relato de diagnóstico médico para câncer, hipertensão, asma, diabete, acidente vascular encefálico (AVE), doença renal, doença da coluna, depressão e doença coronariana (angina e/ou infarto). A residência do participante (morar em Belo Horizonte versus morar em um dos demais municípios da Região Metropolitana de Belo Horizonte) foi também considerada a priori como potencial variável de confusão, objetivando ajustar o modelo para o efeito de possíveis discrepâncias de oferta e acesso aos serviços de saúde entre os municípios que compõem a Região Metropolitana de Belo Horizonte.

\section{Análise dos dados}

As variáveis dependentes "número de consultas domiciliares" e "número de hospitalizações" foram dicotomizadas (sim/não), em razão da pequena proporção de pessoas que tiveram, respectivamente, mais de uma consulta domiciliar e mais de uma hospitalização. O número de consultas médicas foi considerado como variável contagem, e truncada em treze ou mais consultas. Inicialmente, as proporções de usuários dos serviços de saúde (consultas médicas, hospitalização e consulta domiciliar) foram comparadas em função das características predisponentes, capacitantes, de necessidade, além da incapacidade funcional (exposição de interesse), por meio do teste do qui-quadrado de Pearson, com o fator de correção de Rao \& Scott. Análises da associação entre incapacidade funcional e utilização de serviços de saúde foram feitas separadamente para cada uma das variáveis-evento. Essas análises se basearam em razões de prevalências bruta e ajustadas (com respectivos intervalos de $95 \%$ de confiança - IC95\%), estimadas pelos modelos de regressão binomial negativa (para o número de consultas médicas) e de regressão de Poisson com variância robusta (para consultas domiciliares e hospitalizações). No caso do número de consultas médicas, optou-se pelo modelo de regressão binomial negativa em razão da detecção de sobredispersão na distribuição da variável. Isso acontece quando a variância é superior à média, inviabilizando a utilização do modelo de regressão de Poisson ${ }^{18}$. As análises seguiram quatro etapas, correspondentes à introdução sequenciada dos blocos de variáveis independentes, baseadas no modelo de Andersen 16, como se segue: modelo 1 , ajustamento pelas características predisponentes; modelo 2 , acrescentando-se as características capacitantes; modelo 3 (final), acrescentando-se as características de necessidade e o município de residência. Não foi utilizado critério estatístico para deleção de variáveis nos modelos subsequentes, de modo que o modelo final foi ajustado por todas as covariáveis. As variáveis que apresentaram valor de $\mathrm{p}<0,05$ em cada um dos modelos finais (correspondentes a cada um dos serviços de saúde investigados) foram consideradas independentemente associadas às variáveis-evento. As aná- 
lises estatísticas foram realizadas por meio do pacote estatístico Stata, versão 10.0 (Stata Corp., College Station, Estados Unidos). Foram utilizados os procedimentos do programa (comando svy) reservados à análise de dados obtidos em inquéritos populacionais com desenho amostral complexo, que levam em conta o delineamento da amostra e os pesos amostrais dos indivíduos participantes

\section{Aspectos éticos}

O presente estudo foi aprovado pelo Comitê de Ética em Pesquisa da Fundação Oswaldo Cruz, e respeitou os preceitos éticos que regem os experimentos em seres humanos.

\section{Resultados}

Entre os 1.624 participantes do estudo, a idade variou entre 60 e 97 anos (média $=69,7 ; \mathrm{DP}=7,8$ ), com predominância do sexo feminino $(59,6 \%)$, da baixa escolaridade $(73,1 \%$ com escolaridade inferior a 8 anos) e do estado conjugal de casado ou união consensual (52,8\%).

Quanto ao uso de serviços de saúde nos últimos 12 meses, 92,4\%; (IC95\%: 91,0-93,8) dos idosos haviam tido pelo menos uma consulta médica (média = 4,9 consultas; IC95\%; 4,55,2), 4\% (IC95\%: 3,0-5,0) haviam tido uma ou mais consultas domiciliares e 14,3\% (IC95\%:
12,4-16,1) haviam tido uma ou mais internações hospitalares.

Em relação à capacidade funcional, $64,2 \%$ (IC95\%: 61,6-66,9) dos idosos eram independentes para realizar todas as AIVD e ABVD, 19,6\% (IC95\%: 17,4-21,8) apresentavam alguma dificuldade para realizar pelo menos uma AIVD e 16,2\% (IC95\%: 14,2-18,1) tinham alguma dificuldade para realizar uma ou mais ABVD. Todos os idosos com algum grau de dificuldade para pelo menos uma ABVD também o foram para pelo menos uma AIVD, de forma que a prevalência de incapacidade funcional menos grave foi duas vezes maior que a mais grave.

A Tabela 1 apresenta a prevalência de incapacidade para cada uma das diferentes atividades de vida diária (básicas e instrumentais), de acordo com a faixa etária dos participantes. Entre as ABVD, a dificuldade para levantar-se da cama foi a mais frequente $(13,5 \%)$, seguida daquelas para vestir-se $(10,9 \%)$ e para mover-se entre cômodos da casa $(10,3 \%)$; entre as atividades instrumentais, as limitações para realização de tarefas domésticas $(27,9 \%)$ e para fazer compras $(22,1 \%)$ foram as mais relatadas. Em geral, observou-se um aumento na prevalência de limitações para ABVD e AIVD com a idade.

$\mathrm{Na}$ Tabela 2, são apresentados os resultados das análises univariadas da associação entre a capacidade funcional, as características predisponentes, capacitantes e de necessidades de saúde e as variáveis de utilização de serviços de

Tabela 1

Distribuição (\%) das atividades básicas (ABVD) e instrumentais (AIVD) de vida diária segundo tipo de atividade e idade. Região Metropolitana de Belo Horizonte, Minas Gerais, Brasil, 2003.

\begin{tabular}{|c|c|c|c|c|c|c|}
\hline \multirow[t]{2}{*}{ Tipo de atividade } & \multicolumn{5}{|c|}{ Faixa etária (anos) } & \multirow[t]{2}{*}{ Total } \\
\hline & $60-64$ & $65-69$ & $70-74$ & $75-79$ & $80+$ & \\
\hline \multicolumn{7}{|l|}{$A B \vee D$} \\
\hline Levantar-se da cama & 6,8 & 10,6 & 11,9 & 17,4 & 35,1 & 13,5 \\
\hline Vestir-se & 5,2 & 8,1 & 8,0 & 18,7 & 27,8 & 10,9 \\
\hline Mover-se entre cômodos da casa & 3,8 & 7,1 & 8,7 & 16,1 & 30,0 & 10,3 \\
\hline Tomar banho & 3,5 & 6,2 & 5,9 & 12,6 & 22,5 & 8,1 \\
\hline Ir ao banheiro & 3,6 & 5,2 & 3,6 & 10,8 & 21,4 & 7,1 \\
\hline Comer & 2,7 & 4,6 & 3,6 & 10,2 & 17,3 & 6,1 \\
\hline \multicolumn{7}{|l|}{ AIVD } \\
\hline Realizar tarefas domésticas & 13,5 & 24,5 & 28,5 & 42,3 & 56,5 & 27,9 \\
\hline Fazer compras & 9,5 & 16,6 & 21,5 & 32,0 & 56,7 & 22,1 \\
\hline Preparar a comida & 6,7 & 12,2 & 16,3 & 20,1 & 38,0 & 15,4 \\
\hline Administrar o próprio dinheiro & 4,7 & 10,8 & 9,7 & 16,8 & 30,6 & 11,9 \\
\hline Tomar remédio & 3,6 & 6,3 & 4,4 & 13,4 & 24,9 & 8,3 \\
\hline
\end{tabular}


Distribuição percentual (\%) das características sociodemográficas, de condições de saúde e de capacidade funcional segundo a utilização dos serviços de saúde. Região Metropolitana de Belo Horizonte, Minas Gerais, Brasil, 2003.

\begin{tabular}{|c|c|c|c|c|c|c|c|c|}
\hline \multirow[t]{3}{*}{ Características } & \multicolumn{8}{|c|}{ Utilização de serviços de saúde } \\
\hline & \multicolumn{4}{|c|}{ Consulta médica } & \multicolumn{2}{|c|}{ Hospitalização } & \multicolumn{2}{|c|}{ Consulta domiciliar } \\
\hline & $0-1$ & $2-4$ & $5+$ & Valor de $p$ & Sim & Valor de $p$ & Sim & Valor de $p$ \\
\hline \multicolumn{9}{|l|}{ Incapacidade funcional } \\
\hline Não & 35,9 & 37,1 & 27,1 & $<0,001$ & 9,6 & $<0,001$ & 1,2 & $<0,001$ \\
\hline Sim, para AIVD & 18,9 & 40,6 & 40,5 & & 20,3 & & 2,7 & \\
\hline Sim, para ABVD & 13,7 & 35,8 & 50,5 & & 25,6 & & 16,9 & \\
\hline \multicolumn{9}{|l|}{ Sexo } \\
\hline Masculino & 40,3 & 32,1 & 27,6 & $<0,001$ & 14,9 & 0,803 & 2,9 & 0,099 \\
\hline Feminino & 21,0 & 41,3 & 37,6 & & 14,4 & & 4,6 & \\
\hline \multicolumn{9}{|l|}{ Idade (anos) } \\
\hline $60-79$ & 33,2 & 37,9 & 28,9 & $<0,001$ & 13,2 & 0,182 & 2,1 & $<0,001$ \\
\hline $70-79$ & 25,5 & 34,7 & 39,9 & & 15,8 & & 4,7 & \\
\hline $80+$ & 18,2 & 43,1 & 38,8 & & 18,0 & & 10,1 & \\
\hline \multicolumn{9}{|l|}{ Situação conjugal } \\
\hline Casado(a)/União consensual & 31,1 & 38,2 & 30,1 & 0,042 & 15,0 & 0,661 & 3,7 & 0,308 \\
\hline Não casado & 26,5 & 36,8 & 36,8 & & 14,1 & & 4,7 & \\
\hline \multicolumn{9}{|l|}{ Escolaridade (anos de frequência à escola) } \\
\hline $0-3$ & 27,8 & 36,0 & 36,2 & $<0,001$ & 15,5 & 0,247 & 5,9 & 0,001 \\
\hline $4-7$ & 25,1 & 35,7 & 39,2 & & 15,6 & & 1,8 & \\
\hline $8+$ & 35,3 & 41,9 & 22,9 & & 12,0 & & 3,2 & \\
\hline \multicolumn{9}{|l|}{ Filiação a plano de saúde } \\
\hline Não & 32,4 & 33,5 & 34,1 & 0,001 & 14,2 & 0,612 & 4,0 & 0,653 \\
\hline Sim & 24,7 & 42,3 & 33,0 & & 15,1 & & 4,4 & \\
\hline \multicolumn{9}{|l|}{ Número de doenças crônicas } \\
\hline 0 & 45,4 & 37,8 & 16,8 & $<0,001$ & 7,2 & $<0,001$ & 3,2 & 0,173 \\
\hline 1 & 31,3 & 36,4 & 32,4 & & 13,6 & & 3,5 & \\
\hline $2+$ & 14,0 & 38,0 & 48,0 & & 21,3 & & 5,5 & \\
\hline \multicolumn{9}{|l|}{ Autoavaliação da saúde } \\
\hline Muito boa/Boa & 43,3 & 35,9 & 20,8 & $<0,001$ & 7,9 & $<0,001$ & 1,8 & $<0,001$ \\
\hline Razoável & 17,6 & 43,5 & 39,0 & & 17,4 & & 4,5 & \\
\hline Ruim/Muito ruim & 8,3 & 25,7 & 66,0 & & 31,8 & & 12,1 & \\
\hline \multicolumn{9}{|l|}{ Município de residência } \\
\hline $\begin{array}{l}\text { Demais municípios da Região Metropolitana } \\
\text { de Belo Horizonte }\end{array}$ & 33,0 & 31,4 & 35,6 & 0,003 & 16,0 & 0,183 & 3,2 & 0,235 \\
\hline Belo Horizonte & 27,1 & 40,6 & 32,4 & & 13,4 & & 4,4 & \\
\hline
\end{tabular}

ABVD: atividades básicas da vida diária; AIVD: atividades instrumentais da vida diária.

saúde. A incapacidade funcional apresentou-se associada $(\mathrm{p}<0,05)$ a todas as características de utilização de serviços de saúde abordadas. Todas as características apresentaram-se significativamente $(p<0,05)$ associadas à realização de consultas médicas, ao passo que somente características de necessidade (número de doenças crônicas e autoavaliação da saúde foram associadas $(\mathrm{p}<0,05)$ à hospitalização). Já no tocante à consulta domiciliar, o sexo e a idade (entre as predis- ponentes), a escolaridade (entre as capacitantes) e a autoavaliação da saúde (entre as de necessidade) mostraram-se associadas ( $\mathrm{p}<0,05)$.

Os resultados das análises multivariadas da associação entre incapacidade funcional e o número de consultas médicas encontram-se descritos na Tabela 3. Após o ajustamento pelo bloco de variáveis de necessidade, as associações com limitação para as AIVD e para as ABVD, observadas na análise univariada, não se mantiveram 
Tabela 3

Resultados das análises univariada e multivariada da associação entre incapacidade funcional e número de consultas médicas. Região Metropolitana de Belo Horizonte, Minas Gerais, Brasil, 2003.

\begin{tabular}{|c|c|c|c|c|}
\hline Variáveis & RP bruta (IC95\%) & $\begin{array}{c}\text { Modelo } 1 \\
\text { RP ajustada (IC95\%) }\end{array}$ & $\begin{array}{c}\text { Modelo } 2 \\
\text { RP ajustada (IC95\%) }\end{array}$ & $\begin{array}{c}\text { Modelo } 3 \\
\text { RP ajustada (IC95\%) }\end{array}$ \\
\hline \multicolumn{5}{|c|}{ Incapacidade funcional (referência: não) } \\
\hline Sim, para AIVD & $1,40(1,24-1,59)$ & $1,35(1,19-1,54)$ & $1,31(1,16-1,49)$ & $1,07(0,94-1,21)$ \\
\hline Sim, para ABVD & $1,66(1,48-1,85)$ & $1,61(1,43-1,81)$ & $1,57(1,39-1,77)$ & $1,12(0,99-1,27)$ \\
\hline \multicolumn{5}{|l|}{ Sexo (referência: masculino) } \\
\hline Feminino & $1,31(1,18-1,46)$ & $1,26(1,13-1,42)$ & $1,24(1,10-1,38)$ & $1,16(1,03-1,30)$ \\
\hline \multicolumn{5}{|l|}{ Idade [anos] (referência: 60-69) } \\
\hline $70-79$ & $1,23(1,10-1,36)$ & $1,15(1,03-1,28)$ & $1,15(1,03-1,28)$ & $1,13(1,02-1,26)$ \\
\hline $80+$ & $1,25(1,08-1,45)$ & $1,02(0,88-1,17)$ & $1,00(0,87-1,15)$ & $1,13(0,98-1,31)$ \\
\hline \multicolumn{5}{|c|}{$\begin{array}{l}\text { Situação conjugal (referência: casado(a)/união } \\
\text { consensual) }\end{array}$} \\
\hline Não casado & $1,13(1,03-1,25)$ & $0,98(0,87-1,09)$ & $0,97(0,87-1,08)$ & $1,00(0,90-1,11)$ \\
\hline \multicolumn{5}{|c|}{ Escolaridade [anos de frequência à escola] } \\
\hline $4-7$ & $1,02(0,92-1,14)$ & & $1,02(0,91-1,15)$ & $1,04(0,93-1,16)$ \\
\hline $8+$ & $0,75(0,66-0,86)$ & & $0,79(0,69-0,91)$ & $0,92(0,81-1,06)$ \\
\hline \multicolumn{5}{|c|}{ Filiação a plano de saúde (referência: não) } \\
\hline $\operatorname{Sim}$ & $1,04(0,94-1,14)$ & & $1,12(1,01-1,25)$ & $1,17(1,06-1,29)$ \\
\hline Número de doenças crônicas & $1,29(1,24-1,34)$ & & & $1,16(1,12-1,21)$ \\
\hline \multicolumn{5}{|c|}{ Autoavaliação da saúde (referência: muito boa/boa) } \\
\hline Razoável & $1,70(1,53-1,89)$ & & & $1,50(1,34-1,68)$ \\
\hline Ruim/Muito ruim & $2,51(2,22-2,83)$ & & & $2,00(1,73-2,32)$ \\
\hline \multicolumn{5}{|c|}{ Morar em Belo Horizonte (referência: não) } \\
\hline Sim & $0,97(0,88-1,08)$ & & & $1,03(0,92-1,14)$ \\
\hline
\end{tabular}

ABVD: atividades básicas da vida diária; AIVD: atividades instrumentais da vida diária; IC95\%: intervalo de 95\% de confiança; Modelo 1: ajustado pelas características predisponentes (sexo, idade, situação conjugal); Modelo 2: Modelo 1 ajustado pelas características capacitantes (escolaridade, filiação a plano de saúde); Modelo 3: Modelo 2 ajustado pelas características de necessidade (número de doenças crônicas e autoavaliação da saúde) e pelo município de residência (Belo Horizonte versus outros municípios da Região Metropolitana de Belo Horizonte em conjunto); RP: razão de prevalência estimada pelo modelo de regressão binomial negativa.

Nota: o modelo final incluiu 1.590 participantes.

significativas. Entre as variáveis utilizadas para ajustamento do modelo, permaneceram independentemente associadas a um maior número de consultas médicas o sexo feminino, idade (restrita à faixa 70-79 anos), a filiação a plano de saúde e as descritoras da condição de saúde (número de condições crônicas e autoavaliação da saúde).

Entre os eventos investigados, a incapacidade funcional esteve mais fortemente associada à consulta domiciliar, especialmente aquela para ABVD (RP = 8,54; IC95\%: 4,22-17,27). A introdução dos diferentes blocos de variáveis diluiu de forma marcante a força de associação entre limitação para ABVD e consulta domiciliar, detectada na modelagem univariada. Entre as variáveis utilizadas para ajustamento, mantiveram-se inde- pendentemente associadas à consulta domiciliar a idade (apenas a faixa etária de 80 ou mais anos), a escolaridade (no caso, somente a faixa de 4-7 anos) e a pior autoavaliação da saúde (ruim/muito ruim). A consulta domiciliar não foi diferenciada entre os idosos com e sem limitação para a realização de alguma AIVD, embora tenha sido mais frequente entre os primeiros (Tabela 4).

Os resultados dos modelos de análise da associação entre incapacidade funcional e histórico de hospitalização se encontram descritos na Tabela 5. Tanto a limitação para AIVD (RP = 1,62; IC95\%: 1,16-2,26) quanto para ABVD (RP = 1,73; IC95\%: 1,24-2,42) apresentaram-se positiva e independentemente associadas ao histórico de internação hospitalar nos últimos 12 meses. Após a introdução das variáveis descritoras das 
Resultados das análises univariada e multivariada da associação entre incapacidade funcional e as consultas domiciliares. Região Metropolitana de Belo Horizonte, Minas Gerais, Brasil, 2003.

\begin{tabular}{|c|c|c|c|c|}
\hline Variáveis & RP bruta (IC95\%) & $\begin{array}{c}\text { Modelo } 1 \\
\text { RP ajustada (IC95\%) }\end{array}$ & $\begin{array}{c}\text { Modelo } 2 \\
\text { RP ajustada (IC95\%) }\end{array}$ & $\begin{array}{c}\text { Modelo } 3 \\
\text { RP ajustada (IC95\%) }\end{array}$ \\
\hline \multicolumn{5}{|c|}{ Incapacidade funcional (referência: não) } \\
\hline Sim, para AIVD & $2,33(0,90-6,03)$ & $1,92(0,75-4,90)$ & $1,87(0,75-4,70)$ & $1,67(0,69-4,07)$ \\
\hline Sim, para ABVD & $14,52(7,39-28,56)$ & $10,88(5,25-22,53)$ & $10,74(5,27-21,91)$ & $8,54(4,23-17,27)$ \\
\hline \multicolumn{5}{|l|}{ Sexo (referência: masculino) } \\
\hline Feminino & $1,89(1,02-3,51)$ & $1,50(0,80-2,82)$ & $1,37(0,73-2,56)$ & $1,33(0,71-2,49)$ \\
\hline \multicolumn{5}{|l|}{ Idade [anos] (referência: 60-69) } \\
\hline $70-79$ & $2,51(1,37-4,59)$ & $1,79(0,98-3,30)$ & $1,82(0,98-3,38)$ & $1,79(0,96-3,34)$ \\
\hline $80+$ & $5,48(2,55-10,83)$ & $2,35(1,21-4,59)$ & $2,10(1,07-4,12)$ & $2,02(1,03-3,96)$ \\
\hline \multicolumn{5}{|c|}{$\begin{array}{l}\text { Situação conjugal (referência: casado(a)/união } \\
\text { consensual) }\end{array}$} \\
\hline Não casado & $1,27(0,76-2,13)$ & $0,76(0,45-1,26)$ & $0,81(0,48-1,37)$ & $0,89(0,53-1,48)$ \\
\hline \multicolumn{5}{|c|}{ Escolaridade [anos de frequência à escola] } \\
\hline \multicolumn{5}{|l|}{ (referência: 0-3) } \\
\hline $4-7$ & $0,34(0,18-0,66)$ & & $0,36(0,19-0,69)$ & $0,36(0,19-0,68)$ \\
\hline $8+$ & $0,53(0,26-1,05)$ & & $0,72(0,35-1,47)$ & $0,74(0,39-1,41)$ \\
\hline \multicolumn{5}{|c|}{ Filiação a plano de saúde (referência: não) } \\
\hline Sim & $1,13(0,67-1,90)$ & & $1,39(0,85-2,30)$ & $1,40(0,86-2,29)$ \\
\hline Número de doenças crônicas & $1,28(1,05-1,55)$ & & & $0,95(0,75-1,20)$ \\
\hline \multicolumn{5}{|c|}{ Autoavaliação da saúde (referência: muito boa/boa) } \\
\hline Razoável & $2,47(1,29-4,72)$ & & & $1,71(0,92-3,16)$ \\
\hline Ruim/Muito ruim & $6,40(3,20-12,79)$ & & & $2,40(1,26-4,56)$ \\
\hline \multicolumn{5}{|c|}{ Morar em Belo Horizonte (referência: não) } \\
\hline Sim & $1,38(0,81-2,36)$ & & & $1,50(0,84-2,67)$ \\
\hline
\end{tabular}

ABVD: atividades básicas da vida diária; AIVD: atividades instrumentais da vida diária; IC95\%: intervalo de 95\% de confiança; Modelo 1: ajustado pelas características predisponentes (sexo, idade, situação conjugal); Modelo 2: Modelo 1 ajustado pelas características capacitantes (escolaridade, filiação a plano de saúde); Modelo 3: Modelo 2 ajustado pelas características de necessidade (número de doenças crônicas e autoavaliação da saúde) e por município de residência (Belo Horizonte versus outros municípios da Região Metropolitana de Belo Horizonte em conjunto); RP: razão de prevalência estimada pelo modelo de regressão de Poisson, com variância robusta.

Nota: o modelo final incluiu 1.613 participantes.

condições de saúde, nota-se uma atenuação das forças de associação entre incapacidade e hospitalização. Além disso, elas foram as únicas variáveis de ajuste independentemente associadas à internação hospitalar.

\section{Discussão}

Os resultados mais importantes deste trabalho foram: (a) pouco mais de um terço dos idosos apresentou algum tipo de incapacidade funcional, sendo a forma leve (somente para AIVD) mais frequente do que a grave (para ABVD); (b) para todas as atividades pesquisadas, essa condição apresentou uma tendência de aumento com a idade; e (c) o grau mais avançado de limitação
(ABVD) não se mostrou associada à consulta médica, sendo mais fortemente associado à consulta domiciliar do que à hospitalização.

A prevalência global de incapacidade funcional (considerando AIVD e/ou ABVD) verificada em nosso estudo $(35,8 \%)$ foi inferior à encontrada no Sul do Brasil (40\%) 19 e na Espanha $(53,5 \%) 20$, mas superior à descrita em diferentes cenários internacionais 5,21. Se focarmos exclusivamente a limitação para ABVD, a prevalência aqui observada foi maior que a detectada nas populações idosas brasileira e inglesa, em inquéritos de saúde de abrangência nacional (respectivamente, $10,7 \%$ e 9,7\%) ${ }^{14}$. Porém, vale ressaltar que a comparação de prevalências em estudos sobre incapacidade funcional é uma tarefa sobremaneira dificultada pelos variados processos 
Tabela 5

Resultados das análises univariada e multivariada da associação entre incapacidade funcional e a hospitalização. Região Metropolitana de Belo Horizonte, Minas Gerais, Brasil, 2003.

\begin{tabular}{|c|c|c|c|c|}
\hline Variáveis & RP bruta (IC95\%) & $\begin{array}{c}\text { Modelo } 1 \\
\text { RP ajustada (IC95\%) }\end{array}$ & $\begin{array}{c}\text { Modelo } 2 \\
\text { RP ajustada (IC95\%) }\end{array}$ & $\begin{array}{c}\text { Modelo } 3 \\
\text { RP ajustada (IC95\%) }\end{array}$ \\
\hline \multicolumn{5}{|c|}{ Incapacidade funcional (referência: não) } \\
\hline Sim, para AIVD & $2,12(1,55-2,89)$ & $2,20(1,59-3,04)$ & $2,16(1,56-3,00)$ & $1,62(1,16-2,26)$ \\
\hline Sim, para ABVD & $2,67(1,99-3,60)$ & $2,81(2,06-3,82)$ & $2,77(2,03-3,80)$ & $1,73(1,24-2,42)$ \\
\hline \multicolumn{5}{|l|}{ Sexo (referência: masculino) } \\
\hline Feminino & $0,99(0,76-1,29)$ & $0,89(0,67-1,20)$ & $0,89(0,66-1,19)$ & $0,80(0,60-1,06)$ \\
\hline \multicolumn{5}{|l|}{ Idade [anos] (referência: 60-69) } \\
\hline $70-79$ & $1,28(0,97-1,69)$ & $1,09(0,82-1,45)$ & $1,08(0,81-1,44)$ & $1,11(0,84-1,46)$ \\
\hline $80+$ & $1,33(0,90-1,96)$ & $0,90(0,60-1,34)$ & $0,86(0,58-1,27)$ & $1,04(0,71-1,52)$ \\
\hline \multicolumn{5}{|c|}{$\begin{array}{l}\text { Situação conjugal (referência: casado(a)/união } \\
\text { consensual) }\end{array}$} \\
\hline Não casado & $0,98(0,76-1,26)$ & $0,91(0,68-1,22)$ & $0,89(0,67-1,19)$ & $0,92(0,70-1,22)$ \\
\hline \multicolumn{5}{|c|}{ Escolaridade [anos de frequência à escola] } \\
\hline $4-7$ & $0,97(0,73-1,30)$ & & $0,99(0,74-1,32)$ & $1,03(0,77-1,38)$ \\
\hline $8+$ & $0,73(0,51-1,03)$ & & $0,80(0,55-1,17)$ & $1,00(0,68-1,47)$ \\
\hline \multicolumn{5}{|c|}{ Filiação a plano de saúde (referência: não) } \\
\hline $\operatorname{Sim}$ & $1,00(0,77-1,30)$ & & $1,12(0,85-1,47)$ & $1,20(0,91-1,56)$ \\
\hline Número de doenças crônicas & $1,38(1,28-1,50)$ & & & $1,20(1,09-1,32)$ \\
\hline \multicolumn{5}{|c|}{ Autoavaliação da saúde (referência: muito boa/boa) } \\
\hline Razoável & $2,24(1,64-3,08)$ & & & $1,76(1,25-2,47)$ \\
\hline Ruim/Muito ruim & $3,77(2,66-5,34)$ & & & $2,37(1,60-3,52)$ \\
\hline \multicolumn{5}{|c|}{ Morar em Belo Horizonte (referência: não) } \\
\hline Sim & $0,84(0,64-1,09)$ & & & $0,91(0,70-1,19)$ \\
\hline
\end{tabular}

ABVD: atividades básicas da vida diária; AIVD: atividades instrumentais da vida diária; IC95\%: intervalo de 95\% de confiança; Modelo 1: ajustado pelas características predisponentes (sexo, idade, situação conjugal); Modelo 2: Modelo 1 ajustado pelas características capacitantes (escolaridade, filiação a plano de saúde); Modelo 3: Modelo 2 ajustado pelas características de necessidade (número de doenças crônicas e autoavaliação da saúde) e por município de residência (Belo Horizonte versus outros municípios da Região Metropolitana de Belo Horizonte em conjunto); RP: razão de prevalência estimada pelo modelo de regressão de Poisson, com variância robusta.

Nota: o modelo final incluiu 1.609 participantes.

de mensuração utilizados 4 . Alguns estudos medem a incapacidade funcional com base em uma única atividade 22,23 , ao passo que várias atividades são consideradas em outros trabalhos 4,7,14. A incapacidade funcional costuma ser definida em função do grau de dificuldade para realização de atividades, podendo vincular-se a um grau mínimo de dificuldade 4,24,25 ou à total impossibilidade de realizar alguma atividade sem ajuda de terceiros 19; ela pode ainda ser medida em termos do número de atividades em algum grau comprometidas $4,7,20$ ou pelo estabelecimento de um escore numérico resultante do total de atividades para as quais o indivíduo apresenta restrições para sua realização ${ }^{8}$.

Diante disso, a opção pela medida do comprometimento funcional mediante "qualquer di- ficuldade" na realização da atividade considerada objetivou uma maior sensibilidade na coleta da informação. A intenção foi dimensionar mais precocemente a incapacidade funcional, permitindo aquilatar suas repercussões, antes que um processo de incapacidade grave se estabeleça, favorecendo assim a efetividade das ações dos serviços de saúde na população assistida. Provavelmente, a prevalência da incapacidade funcional encontrada em nosso estudo tenderá a ser mais elevada do que a mensurada em pesquisas que utilizaram um critério mais específico para classificação da incapacidade (vinculado a um maior número de atividades com incapacidade ou a um maior grau de dependência na realização delas). A superação dessas dificuldades na comparação de resultados observados em diferentes popula- 
ções - mister da pesquisa epidemiológica - passa necessariamente pelo estabelecimento de critérios mais homogêneos na definição da incapacidade funcional 20 .

Estudos internacionais 21,26 e nacionais 19,24 têm mostrado que a limitação para as AIVD se apresenta mais frequente que a limitação para ABVD, independentemente dos procedimentos adotados na sua mensuração. Nossos resultados corroboram a literatura, pois entre os idosos da Região Metropolitana de Belo Horizonte, a proporção de idosos com limitações para AIVD foi superior àquela com limitações para ABVD, evidenciando que a dificuldade em realizar atividades mais elaboradas e de maior complexidade responde pela maior parcela da incapacidade funcional encontrada nessa população. Cabe ressaltar que o processo de incapacidade é dinâmico, e, em sua progressão, a limitação em atividades mais fortemente relacionadas à vida social antecede aquela relacionada a atividades de autocuidado e de sobrevivência 3 , e a dependência em alguma AIVD sabidamente predispõe ao comprometimento em ABVD 27. Esse curso de progressão da incapacidade tem implicações importantes para a saúde pública, visto que o reconhecimento e a prevenção dos fatores de risco, bem como o cuidado em caso de detecção de alguma incapacidade para a AIVD, representam etapas essenciais à prevenção da limitação futura na realização das ABVD e às potenciais conse quências nefastas disso ao indivíduo, às famílias e ao sistema de saúde.

Olhando especificamente para as diferentes atividades investigadas, as maiores prevalências de limitação foram observadas para realização de tarefas domésticas e de compras, atividades que demandam, respectivamente, maiores esforços físico e cognitivo. Spillman ${ }^{5}$ evidenciou a contribuição da redução da incapacidade para AIVD na redução da incapacidade total entre idosos norte-americanos. Uma parte da redução da incapacidade para algumas atividades instrumentais (como manejar dinheiro e fazer compras) pode ser obtida por meio de mudanças ambientais e tecnológicas, mas essa redução não significará qualquer impacto positivo na prevenção da incapacidade para ABVD 5. Por sua vez, a baixa prevalência observada para a limitação em alimentar-se encontra eco na literatura 5,20,23 e representa alto grau de dependência e forte marcador de mortalidade. Nossos resultados se assemelham àqueles encontrados em um estudo realizado no Sul do Brasil, que entre diversas atividades avaliadas, encontrou menores frequências de restrições para as atividades de comer, deitar/levantar-se da cama e ir ao banheiro 19 . Vale destacar que a análise específica por ativida- de evidencia o grau de consistência interna dos dados, na medida em que, nessa população, a limitação para AIVD foi mais comum que aquela para ABVD.

A idade é sabidamente um importante determinante da incapacidade funcional: os idosos experimentam mais intensamente essa condição de saúde em comparação aos adultos e jovens e, no grupo idoso, indivíduos mais velhos tendem a ser menos independentes que os mais jovens, existindo uma vasta documentação de evidências da associação entre tais variáveis em pesquisas internacionais 7,21 e no Brasil 22,23,25. Nosso estudo corrobora esses achados, uma vez que todas as atividades investigadas apresentaram um padrão consistente com o aumento da incapacidade funcional em função do avanço da idade.

Na comparação entre os idosos com prejuízo funcional mais leve (para realização das AIVD) e os independentes, os primeiros utilizaram mais os serviços de saúde (consulta médica, consulta domiciliar e hospitalização), mas, após o ajustamento pelas covariáveis do estudo, apenas a hospitalização se manteve independentemente associada. Associação positiva entre incapacidade para AIVD e hospitalização também foi encontrada entre idosos espanhóis 20 , enquanto a ausência de associação entre consulta médica e o mesmo tipo de incapacidade foi verificada em idosos israelenses 28 . Como os serviços de atenção primária são mais dependentes da percepção do paciente sobre sua saúde 13 , é possível que a limitação ou incapacidade para AIVD não seja compreendida como motivo para demandar uma consulta médica; ou ainda que a limitação física relacionada às AIVD pesquisadas restrinja o acesso a (e o excesso de) consultas médicas 28.

As associações entre limitações para ABVD e utilização de serviços de saúde, independentemente da presença das covariáveis, só não permaneceram significativas para a consulta médica. Nossos resultados são consistentes com os de outros estudos 13,20,27,29. Em relação à associação com hospitalização, é possível que as condições de saúde subjacentes à incapacidade respondam por essa associação positiva 28. Por outro lado, há evidências do frequente envolvimento das internações hospitalares no agravamento da condição funcional da pessoa idosa 30 . O desenho transversal adotado no presente estudo constitui uma limitação importante no estabelecimento da ordem cronológica na ocorrência da exposição e do evento. Assim, não é possível estabelecer se, nessa associação, a incapacidade funcional determina ou é determinada pela hospitalização.

Entre os serviços de saúde considerados no estudo, a consulta domiciliar foi a mais fortemente associada à limitação para ABVD. 
A magnitude da associação entre consulta domiciliar e incapacidade funcional grave é de certa forma esperada porque idosos com esse grau de incapacidade apresentam maiores dificuldades para se deslocar para fora do seu domicílio, requerendo, portanto, um cuidado à saúde em seus próprios domínios 26. A literatura demonstra a efetividade desse serviço em reduzir internações desnecessárias e minimizar a demanda dos serviços de emergência entre os idosos com incapacidade, evitando, por conseguinte, o aumento dos custos dos serviços de saúde; além disso, a satisfação dos usuários e dos cuidadores com esse tipo de serviço confirmam a qualidade da atenção 31. Chama a atenção, no entanto, o fato de que apenas $4 \%$ dos idosos investigados, e, entre esses, apenas 16,9\% daqueles com incapacidade para ABVD tiveram uma consulta domiciliar nos 12 meses anteriores à entrevista, sugerindo dificuldades de acesso a essa modalidade de atendimento.

Considerando o modelo de Andersen 16, as variáveis do bloco de necessidades desempenharam um papel mais marcante no ajustamento da associação entre incapacidade funcional e as distintas modalidades de atenção à saúde, se comparado aos blocos de variáveis predisponentes e capacitantes. Elas foram responsáveis por importantes diluições nas forças de associação entre a incapacidade funcional e o uso de serviços de saúde, além de permaneceram independentemente associadas à utilização de serviços de saúde. A exceção foi o número de condições de saúde, que não permaneceu associado à consulta domiciliar no modelo multivariado. Esses resultados estão de acordo com o verificado em outras populações 28 e indicam a pertinência da utilização dessas variáveis na investigação, numa perspectiva de obtenção de uma maior precisão dos resultados. Ainda em relação às variáveis de ajustamento, os resultados relativos à residência do participante apontam que eventuais diferenças de oferta e acesso a serviços de saúde entre os municípios da Região Metropolitana de Belo Horizonte não tiveram qualquer influência na associação entre incapacidade funcional e utilização de serviços de saúde.

Este estudo tem como vantagens a base populacional estudada, representativa da Região Metropolitana de Belo Horizonte (uma das três maiores do país) e a inclusão de diferentes fontes de serviços de saúde, inclusive a atenção domiciliar, um aspecto emergente da saúde do idoso. Uma possível limitação, comum aos inquéritos domiciliares, é a exclusão de idosos institucionalizados, o que poderia levar a uma subestimativa na prevalência da incapacidade funcional. Entretanto, como menos de $1 \%$ da população idosa belorizontina encontra-se institucionalizada 25 , este fato, provavelmente, não introduziu importantes distorções no estudo.

Os resultados deste trabalho mostram o maior uso de serviços de saúde por idosos com incapacidade funcional, sendo mais forte a associação entre a incapacidade grave e consultas domiciliares. Porém, tal serviço se mostrou inacessível a mais de $80 \%$ dos idosos com incapacidade grave. Por outro lado, a ausência de associação entre incapacidade e consultas médicas, verificada simultaneamente à presença de associação entre elas e doenças crônicas, pode ser um indício de que a atuação dos serviços de saúde ainda permanece primordialmente pautada pelo diagnóstico e tratamento de doenças crônicas, e não pela manutenção e recuperação da capacidade funcional do idoso. Isso contraria a legislação brasileira, que preconiza expressamente a garantia do "atendimento domiciliar para a população idosa que dele necessitar e esteja impossibilitada de se locomover" 32. Faz-se necessário efetivar a implementação de políticas pró-envelhecimento ativo para toda a população, idosa e não idosa, como forma de prevenir a incapacidade funcional. Além disso, é preciso ampliar o acesso aos serviços de saúde para o segmento populacional idoso, com a inclusão de ações de reabilitação de complexidade variável, de modo a reduzir a incapacidade e a consequente necessidade (atual e futura) de utilização de serviços de saúde, especialmente os mais complexos e onerosos, como a hospitalização e as consultas domiciliares. 


\section{Resumen}

Este estudio investigó el patrón de asociación entre la capacidad funcional y el uso de los servicios de salud entre los ancianos que viven en el área metropolitana de Belo Horizonte, Minas Gerais, Brasil. El estudio incluyó a 1.624 pacientes ancianos ( $\geq 60$ años), seleccionados por muestreo aleatorio. La variable dependiente fue el uso de los servicios de salud, sobre la base de tres descriptores: número de visitas al médico, visitas a domicilio y hospitalizaciones. La variable independiente fue la capacidad funcional, definida por la dificultad informada en la realización de actividades básicas de la vida diaria (ABVD) y actividades instrumentales de la vida diaria (AIVD). La dificultad en la realización de AIVD sólo se asoció con la hospitalización ( $R P=1,62$; IC95\%: 1,16-2,26), mientras que la limitación funcional en actividades de la vida diaria se presenta asociada a la hospitalización ( $R P=1,73$; IC95\%: 1,24-2,42) y a la consulta en casa ( $R P=8,54 ;$ IC95\%: 4,22-17,27). Estos resultados apuntan una mayor utilización de los servicios de salud entre los adultos mayores con limitaciones funcionales, especialmente en los más caros, como la hospitalización y consulta en casa, y que la dimensión funcional no ha guiado estos servicios, aún condicionados por la presencia de enfermedades.

Servicios de Salud; Anciano Frágil; Anciano

\section{Colaboradores}

C. B. Fialho concebeu o projeto, analisou os dados e interpretou os resultados e redigiu o artigo. M. F. LimaCosta colaborou na concepção do projeto, na redação e revisão crítica do artigo e aprovou sua versão final. K. C. Giacomin participou da redação e da revisão crítica do artigo e aprovou a versão final. A. I. Loyola Filho orientou a concepção do projeto, realizou a análise dos dados e interpretação dos resultados, participou da redação e revisão crítica do artigo e aprovou a versão final.

\section{Agradecimentos}

A autora principal foi bolsista Capes no Programa de Pós-graduação em Ciências da Saúde, do Centro de Pesquisas René Rachou, Fundação Oswaldo Cruz. O Inquérito de Saúde da Região Metropolitana de Belo Horizonte foi desenvolvido como parte das atividades do Núcleo de Estudos em Saúde Pública e Envelhecimento, Fundação Oswaldo Cruz/Universidade Federal de Minas Gerais e financiada pela Secretaria deVigilância à Saúde do Ministério da Saúde e pelo Banco Mundial.

\section{Referências}

1. Wong LLR, Carvalho JA. O rápido processo de envelhecimento populacional do Brasil: sérios desafios para as políticas públicas. Rev Bras Estud Popul 2006; 23:5-26.

2. Schramm JMA, Oliveira AF, Leite IC, Valente JG, Gadelha AMJ, Portela MC, et al. Transição epidemiológica e o estudo de carga de doença no Brasil. Ciênc Saúde Coletiva 2004; 9:897-908

3. Verbrugge L, Jette A. The disablement process. Soc Sci Med 1994; 38:1-14.

4. Alves LC, Leite IC, Machado CJ. Conceituando e mensurando a incapacidade funcional da população idosa: uma revisão de literatura. Ciênc Saúde Coletiva 2008; 13:1199-207.

5. Spillman BC. Changes in elderly disability rates and the implications for health care utilization and cost. Milbank Q 2004; 82:157-94.
6. Alves LC, Leimann BCQ, Vasconcelos MEL, Carvalho MS, Vasconcelos AGG, Fonseca TCO, et al. A influência das doenças crônicas na capacidade funcional dos idosos do Município de São Paulo, Brasil. Cad Saúde Pública 2007; 23:1924-30.

7. Reyes-Ortiz C, Ostir G, Pelaez M, Ottenbacher K. Cross-national comparison of disability in Latin American and Caribbean persons aged 75 and older. Arch Gerontol Geriatr 2006; 42:21-33.

8. Rosa TEC, Benício MHD'A, Latorre MRDO, Ramos LR. Fatores determinantes da capacidade funcional entre idosos. Rev Saúde Pública 2003; 37:40-8.

9. Rodrigues MAP, Facchini LA, Piccini RX, Tomasi E, Thumé E, Silveira DS, et al. Uso de serviços básicos de saúde por idosos portadores de condições crônicas, Brasil. Rev Saúde Pública 2009; 43:604-12. 
10. Sawyer DO, Leite IC, Alexandrino R. Perfis de utilização de serviços de saúde no Brasil. Ciênc Saúde Coletiva 2002; 7:757-76.

11. Loyola Filho AI, Matos DL, Giatti L, Afradique ME, Peixoto SV, Lima-Costa MF. Causas de internações hospitalares entre idosos brasileiros no âmbito do Sistema Único de Saúde. Epidemiol Serv Saúde 2004; 13:229-38.

12. Gulley SP, Altman BM. Disability in two health care systems: access, quality, satisfaction, and physician contacts among working-age Canadians and Americans with disabilities. Disabil Health J 2008; 1:196-208

13. Hansen MS, Fink P, Frydenberg M, Oxhoj ML. Use of health services, mental illness, and self-rated disability and health in medical inpatients. Psychosom Med 2002; 64:668-75.

14. Lima-Costa MF, Oliveira C, Macinko J, Marmot M. Socioeconomic inequalities in health in older adults in Brazil and England. Am J Public Health 2012; 102:1535-41.

15. Lima-Costa MFF. A saúde dos adultos na Região Metropolitana de Belo Horizonte: um estudo epidemiológico de base populacional. Belo Horizonte: Núcleo de Estudos em Saúde Pública e Envelhecimento, Fundação Oswaldo Cruz/Universidade Federal de Minas Gerais; 2004.

16. Andersen RM. Revisiting the behavioral model and access to medical care: does it matter? J Health Soc Behav 1995; 36:1-10.

17. Lafortune L, Béland F, Bergman H, Ankri J. Health state profiles and service utilization in community-living elderly. Med Care 2009; 47:286-94.

18. Long JS, Freese J. Regression for categorical dependent variables using Stata. College Station: Stata Press; 2006.

19. Del Duca GF, Silva MC, Hallal PC. Incapacidade funcional para atividades básicas e instrumentais da vida diária em idosos. Rev Saúde Pública 2009; 43:796-805.

20. Millán-Calenti JC, Tubío J, Pita-Fernández S, González-Abraldes I, Lorenzo T, Fernández-Arruty $\mathrm{T}$, et al. Prevalence of functional disability in activities of daily living (ADL), instrumental activities of daily living (IADL) and associated factors, as predictors of morbidity and mortality. Arch Gerontol Geriatr 2010; 50:306-10

21. Gureje O, Ogunniyi A, Kola L, Afolabi E. Functional disability in elderly Nigerians: results from the Ibadan Study of Aging. J Am Geriatr Soc 2006; 54:1784-9.
22. Alves LC, Leite IC, Machado CJ. Fatores associados à incapacidade funcional dos idosos no Brasil: análise multinível. Rev Saúde Pública 2010; 44:468-78.

23. Parahyba MI, Veras R. Diferenciais sociodemográficos no declínio funcionais em mobilidade física entre os idosos no Brasil. Ciênc Saúde Coletiva 2008; 13:1257-64.

24. Maciel ACC, Guerra RO. Influência dos fatores biopsicossociais sobre a capacidade funcional de idosos residentes no nordeste do Brasil. Rev Bras Epidemiol 2007; 10:178-89.

25. Giacomin KC, Peixoto SV, Uchoa E, Lima-Costa M. Estudo de base populacional dos fatores associados à incapacidade funcional entre idosos na Região Metropolitana de Belo Horizonte, Minas Gerais, Brasil. Cad Saúde Pública 2008; 24:1260-70.

26. Rochat S, Cumming RG, Blyth F, Creasey H, Handelsman D, Le Couteur DG, et al. Frailty and use of health and community services by community-dwelling older men: the Concord Health and Ageing in Men Project. Age Ageing 2010; 39:228-33.

27. Hardy SE, Gill TM. Recovery from disability among community-dwelling older persons. JAMA 2004; 291:1596-602.

28. Walter-Ginzburg A, Chetrit A, Medina C, Blumstein T, Gindin J, Modan B. Physician visits, emergency room utilization, and overnight hospitalization in the old-old in Israel: the Cross-sectional and Longitudinal Aging Study (CALAS). J Am Geriatr Soc 2001; 49:549-56.

29. Gill TM, Allore H, Holford TR, Guo Z. The development of insidious disability in activities of daily living among community-living older persons. Am J Med 2004; 117:484-91.

30. Giacomin KC, Couto EC. A fiscalização das ILPIS: o papel dos conselhos, do Ministério Público e da Vigilância Sanitária. In: Camarano AA, organizadora. Cuidados de longa duração para a população idosa: um novo risco social a ser assumido? Brasília: Instituto de Pesquisa Econômica Aplicada; 2010. p. 213-48.

31. Béland F, Bergman H, Lebel P, Clarfield M, Tousignant $\mathrm{P}$, Contandriopoulos AP, et al. A system of integrated care for older persons with disabilities in Canada: results from a randomized controlled trial. J Gerontol A Biol Sci Med Sci 2006; 61:367-73.

32. Presidência da República. Lei no 10.741 , de 1o de outubro de 2003. Estatuto do idoso. Diário Oficial da União 2003; 3 out.

Recebido em 24/Abr/2013

Versão final reapresentada em 19/Jul/2013

Aprovado em 27/Ago/2013 